\title{
Poroelasticity of a covalently crosslinked alginate hydrogel under compression
}

\section{Citation}

Cai, Sengqiang, Yuhang Hu, Xuanhe Zhao, and Zhigang Suo. 2010. “Poroelasticity of a Covalently Crosslinked Alginate Hydrogel under Compression." Journal of Applied Physics 108 (11): 113514. https://doi.org/10.1063/1.3517146.

\section{Permanent link}

http://nrs.harvard.edu/urn-3:HUL.InstRepos:41461224

\section{Terms of Use}

This article was downloaded from Harvard University's DASH repository, and is made available under the terms and conditions applicable to Other Posted Material, as set forth at http:// nrs.harvard.edu/urn-3:HUL.InstRepos:dash.current.terms-of-use\#LAA

\section{Share Your Story}

The Harvard community has made this article openly available.

Please share how this access benefits you. Submit a story.

Accessibility 


\title{
Poroelasticity of a covalently crosslinked alginate hydrogel under compression
}

\author{
Sengqiang Cai, ${ }^{1}$ Yuhang Hu, ${ }^{1}$ Xuanhe Zhao, ${ }^{2}$ and Zhigang Suo ${ }^{1, a)}$ \\ ${ }^{1}$ School of Engineering and Applied Sciences, Kavli Institute for Nanobio Science and Technology, \\ Harvard University, Cambridge, Massachusetts 02138, USA \\ ${ }^{2}$ Department of Mechanical Engineering and Materials Science, Duke University, Durham, \\ North Carolina 27708, USA
}

(Received 15 September 2010; accepted 18 October 2010; published online 7 December 2010)

This paper studies the poroelastic behavior of an alginate hydrogel by a combination of theory and experiment. The gel—covalently crosslinked, submerged in water, and fully swollen-is suddenly compressed between two parallel plates. The gap between the plates is held constant subsequently, and the force on the plate relaxes while water in the gel migrates. This experiment is analyzed by using the theory of linear poroelasticity. A comparison of the relaxation curve recorded in the experiment and that derived from the theory determines the elastic constants and the permeability of the gel. The material constants so determined agree well with those determined by using a recently developed indentation method. Furthermore, during relaxation, the concentration of water in the gel is inhomogeneous, resulting in tensile hoop stresses near the edge of the gel, and possibly causing the gel to fracture. (C) 2010 American Institute of Physics. [doi:10.1063/1.3517146]

\section{INTRODUCTION}

A flexible, covalent network of polymers can imbibe a large quantity of a solvent, resulting in a gel. Gels constitute many tissues of animals and plants, and are used in diverse applications, including drug delivery, ${ }^{1,2}$ microfluidics, ${ }^{3,4}$ tissue engineering, ${ }^{5,6}$ oilfield management, ${ }^{7,8}$ and food processing. ${ }^{9,10}$ The mechanical behavior of gels ${ }^{11-13}$ and gellike tissues (e.g., cartilage $)^{14,15}$ is time-dependent. The network enables large and reversible deformation, while the solvent in the gel migrates. The concurrent deformation of the network and migration of the solvent is known as poroelasticity.

We have recently reported experiments on an alginate hydrogel pressed by a flat plate ${ }^{16}$ and by an indenter. ${ }^{17}$ In each experiment, a disk of an alginate hydrogel is covalently crosslinked, submerged in water or aqueous solution, and fully swollen. The gel is pressed by suddenly pressing the plate [Fig. 1(a)] or the indenter [Fig. 1(b)]. The displacement is kept constant subsequently [Fig. 1(c)], while the force on the plate or the indenter is recorded as a function of time [Fig. 1(d)]. The force instantly rises and then relaxes, as water in the gel migrates and the gel approaches a new state of equilibrium. This relaxation curve is used to deduce material constants of the gel-the shear modulus and Poisson's ratio of the gel, as well as the permeability of the solvent through the network.

The main object of this paper is to ascertain that the two methods-compression and indentation-yield the same material constants for the same gel. To minimize the variability of the gel used in the two experiments, here we conduct both experiments by using the alginate hydrogel prepared in the same batch. The material constants of the gel are determined by comparing the relaxation curves obtained from the experi-

${ }^{a)}$ Electronic mail: suo@seas.harvard.edu. ments to those derived from the theory of poroelasticity. Our previous paper ${ }^{17}$ has reported the theoretical relaxation curve for indentation, and this paper will derive the theoretical relaxation curve for compression. Furthermore, we will describe the theoretical prediction of transient fields in the
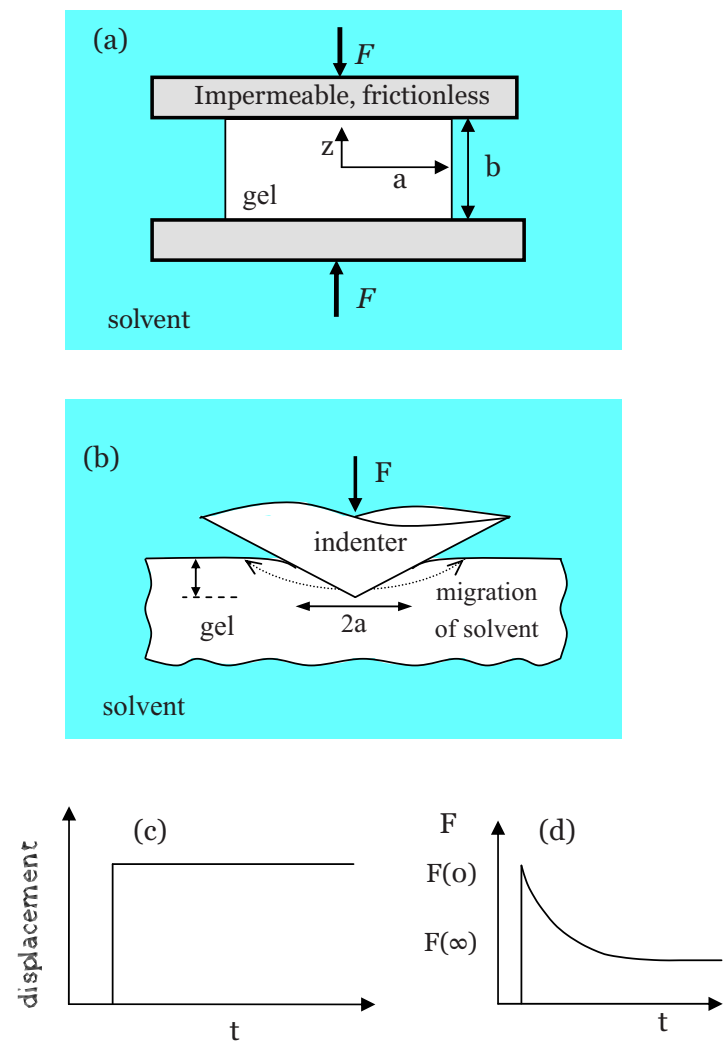

FIG. 1. (Color online) (a) A disk of a gel is submerged in a solvent, and is compressed by frictionless, impermeable, rigid plates. (b) A disk of a gel is submerged in a solvent, and a conical indenter is pressed into the gel. (c) In both experiments, the displacement is suddenly prescribed and subsequently held fixed. (d) The force is recorded as a function of time. 
compressed gel. In particular, the transient hoop stress is tensile near the edge of the gel, and may cause the gel to fracture.

\section{GOVERNING EQUATIONS OF POROELASTICITY}

This section writes Biot's theory of poroelasticity ${ }^{18}$ in a form suitable for the analysis of the compression test. The presentation will be brief; details concerning application of the theory to polymer gels may be found elsewhere (e.g., Refs. 12, 13, 19, and 20). Figure 1 illustrates a disk of a gel, radius $a$ and thickness $b$, along with the cylindrical coordinates $(r, \theta, z)$. The disk is pressed vertically, and the gel is slippery between the two plates, so that the disk is taken to deform under the condition of generalized plane strain. The axial strain is homogeneous in the gel but can vary with time. Let $\varepsilon_{z}(t)$ be the axial strain of the gel as a function of time. The deformation of the disk is taken to be axisymmetric, so that the radial displacement $u$ is independent of $z$ and $\theta$ but is a function of time and radial position. Write the field of the radial displacement as $u(r, t)$. The hoop strain and the radial strain are

$$
\begin{aligned}
& \varepsilon_{\theta}=u / r, \\
& \varepsilon_{r}=\partial u / \partial r .
\end{aligned}
$$

All the shear strains vanish.

The plates are impermeable to the solvent, and the solvent in the gel migrates in the radial direction. Let $J(r, t)$ be the flux of the solvent (i.e., the number of solvent molecules crossing unit area in a reference state per unit time). Let $C(r, t)$ be the field of the concentration (i.e., the number of solvent molecules per unit volume of the gel in the reference state). The number of solvent molecules is conserved:

$$
\frac{\partial C}{\partial t}+\frac{\partial(r J)}{r \partial r}=0 .
$$

The gel is in mechanical equilibrium at all time. The radial stress $\sigma_{r}(r, t)$ and the hoop stress $\sigma_{\theta}(r, t)$ satisfy

$$
\frac{\partial \sigma_{r}}{\partial r}+\frac{\sigma_{r}-\sigma_{\theta}}{r}=0
$$

The axial stress $\sigma_{z}(r, t)$ gives rise to the compressive force:

$$
F(t)=-2 \pi \int_{0}^{a} \sigma_{z} r d r .
$$

We adopt the sign convention that the compressive force $F$ is positive. All components of the shear stresses vanish.

The gel, however, is not in diffusive equilibrium. The chemical potential of the solvent in the gel is a timedependent field $\mu(r, t)$. The gradient of the chemical potential $\partial \mu / \partial r$ drives the flux of the solvent. The two quantities are taken to be linearly related, written in the form

$$
J=-\frac{k}{\eta \Omega^{2}} \frac{\partial \mu}{\partial r},
$$

where $\eta$ is the viscosity of the solvent and $\Omega$ the volume per solvent molecule. Both $\eta$ and $\Omega$ are taken to be the values for the pure liquid solvent (e.g., for water $\eta=1.0$ $\times 10^{-3} \mathrm{~N} \mathrm{~s} \mathrm{~m}^{-2}$ and $\Omega=3.0 \times 10^{-29} \mathrm{~m}^{3}$ ). Consequently, (6) defines a phenomenological quantity, $k$, which is known as the permeability and has the dimension of length squared.

At any time, each differential element of the gel is in a state of thermodynamic equilibrium. A reference state is assigned when the gel is stress-free and the solvent in the gel is in equilibrium with the pure liquid solvent. In the reference state, the strains of the gel are set to be zero, the chemical potential of the solvent in the gel is set to be zero, and the concentration of the solvent in the gel is denoted by $C_{0}$. When the gel is subject to a state of stress, the gel is in another state of equilibrium, in which the gel deforms and the solvent in the gel may no longer be in equilibrium with the pure liquid solvent. This state of equilibrium of the gel is characterized by the stresses $\left(\sigma_{r}, \sigma_{\theta}, \sigma_{z}\right)$, the strains $\left(\varepsilon_{r}, \varepsilon_{\theta}, \varepsilon_{z}\right)$, the concentration $C$, and the chemical potential of the solvent $\mu$. These thermodynamic variables are connected through the equations of state, as described below.

Because the stress in a gel is typically small, the polymers and the solvent molecules are commonly assumed to be incompressible. Consequently, the increase in the volume of the gel is entirely due to the volume of the absorbed solvent:

$$
\varepsilon_{r}+\varepsilon_{\theta}+\varepsilon_{z}=\Omega\left(C-C_{0}\right) .
$$

The gel is assumed to be isotropic, and the stresses are assumed to be linear in strains. Under these assumptions, the equations of state take the form ${ }^{17}$

$$
\begin{aligned}
& \sigma_{r}=2 G\left[\varepsilon_{r}+\frac{\nu}{1-2 \nu}\left(\varepsilon_{r}+\varepsilon_{\theta}+\varepsilon_{z}\right)\right]-\frac{\mu}{\Omega}, \\
& \sigma_{\theta}=2 G\left[\varepsilon_{\theta}+\frac{\nu}{1-2 \nu}\left(\varepsilon_{r}+\varepsilon_{\theta}+\varepsilon_{z}\right)\right]-\frac{\mu}{\Omega}, \\
& \sigma_{z}=2 G\left[\varepsilon_{z}+\frac{\nu}{1-2 \nu}\left(\varepsilon_{r}+\varepsilon_{\theta}+\varepsilon_{z}\right)\right]-\frac{\mu}{\Omega},
\end{aligned}
$$

where $G$ is the shear modulus and $\nu$ Poisson's ratio. When the gel is constrained by rigid and permeable walls in all directions, such that all strains vanish, an increase in the chemical potential of the solvent gives rise to a hydrostatic pressure, $\mu / \Omega$.

The above equations specify the theory of poroelasticity. A combination of these equations gives the governing equations for the fields $C(r, t), u(r, t)$, and $\mu(r, t)$ :

$$
\begin{aligned}
& \frac{\partial(r u)}{r \partial r}+\varepsilon_{z}(t)=\Omega\left(C-C_{0}\right), \\
& \frac{2(1-\nu)}{(1-2 \nu)} \frac{\partial}{\partial r}\left[\frac{\partial(r u)}{r \partial r}\right]=\frac{\partial \mu}{G \Omega \partial r}, \\
& \frac{\partial C}{\partial t}=\frac{D \partial}{r \partial r}\left(\frac{r \partial C}{\partial r}\right),
\end{aligned}
$$

with the diffusivity given by 


$$
D=\frac{2(1-\nu) G k}{(1-2 \nu) \eta}
$$

Equation (13) takes the familiar form of the diffusion equation. In poroelasticity, however, this diffusion equation cannot be solved by itself, because the boundary conditions typically involve the chemical potential and the displacement. Nonetheless, (13) indicates that over time $t$ a disturbance diffuses over a length $\sqrt{D t}$.

Throughout the experiment, the gel is submerged in a pure liquid solvent, whose chemical potential is set to be zero. Before being compressed, the gel is in equilibrium with the external solvent - a state taken to be the reference state of the gel. At time $t=0$, a compressive strain of magnitude $\varepsilon$ is suddenly prescribed by pressing the rigid plates, and this strain is held constant in subsequent time. That is, $\varepsilon_{z}(t)=-\varepsilon$, for $t \geq 0$. We adopt the sign convention that $\varepsilon>0$ for compression.

The boundary conditions on the edge of the disk are obtained by assuming that the gel is locally in equilibrium with the external solvent at all time. Thus, the chemical potential of the solvent in the gel, on the edge, equals that of the external solvent at all time:

$$
\mu(a, t)=0 \text {. }
$$

Furthermore, the radial stress on the edge of the gel vanishes at all time:

$$
\sigma_{r}(a, t)=0 .
$$

Inserting (15) and (16) into (8), we obtain a boundary condition in terms of the displacement:

$$
(1-\nu) \frac{\partial u}{\partial r}(a, t)+\nu\left[\frac{u(a, t)}{r}-\varepsilon\right]=0
$$

\section{SHORT-TIME AND LONG-TIME LIMITS}

The compression causes a portion of the solvent in the gel to migrate out, so that the field in the gel evolves with time. We first consider the short-time limit, $t=0$, instantaneously after the gel is compressed with the strain $\varepsilon$. The gel undergoes a homogeneous deformation. Instantaneously after the gel is compressed, the solvent in the gel has no time to migrate so that $C(r, 0)=C_{0}$, and the volume of the gel does not change, $\varepsilon_{r}+\varepsilon_{\theta}+\varepsilon_{z}=0$. The axial strain is $\varepsilon_{z}=-\varepsilon$, and the radial and hoop strains are

$$
\varepsilon_{r}(r, 0)=\varepsilon_{\theta}(r, 0)=\frac{\varepsilon}{2}
$$

The radial displacement is

$$
u(r, 0)=\frac{1}{2} \varepsilon r .
$$

Instantaneously after compression, the radial and the hoop stresses are zero, $\sigma_{r}(r, 0)=\sigma_{\theta}(r, 0)=0$. The solvent in the gel is out of equilibrium with the external solvent: the chemical potential of the solvent in the gel is homogeneous but is not zero. Setting $\sigma_{r}(r, 0)=0$ and $\varepsilon_{r}+\varepsilon_{\theta}+\varepsilon_{z}=0$ in Eq. (8), we obtain that

$$
\mu(r, 0)=\varepsilon G \Omega \text {. }
$$

From (10) we obtain the axial stress

$$
\sigma_{z}(r, 0)=-3 G \varepsilon .
$$

Recall that the edge of the gel is assumed to be in local equilibrium with the external solvent at all time, so that $\mu(a, 0)=0$ instantaneously after compression. This boundary value is unequal to the value in the interior of the gel, $\mu(r, 0)=\varepsilon G \Omega$. Such a discontinuity is common in initial/ boundary-value problems subject to suddenly prescribed initial conditions. We now examine the consequence of this discontinuity in the chemical potential. Geometric compatibility requires that $\varepsilon_{\theta}(a, 0)=\varepsilon / 2$, while mechanical equilibrium requires that $\sigma_{r}(a, 0)=0$. Inserting these conditions, along with $\mu(a, 0)=0$, into (8) $-(10)$, we obtain the instantaneous radial strain

$$
\varepsilon_{r}(a, 0)=\frac{\nu \varepsilon}{2(1-\nu)},
$$

the hoop stress

$$
\sigma_{\theta}(a, 0)=\frac{1-2 \nu}{1-\nu} G \varepsilon,
$$

and the axial stress

$$
\sigma_{z}(a, 0)=-\frac{2-\nu}{1-\nu} G \varepsilon
$$

The radial strain on the edge (22) differs from that in the interior of the gel, $\varepsilon_{r}(r, 0)=\varepsilon / 2$. Similarly, the hoop and axial stresses also differ from their counterparts in the interior of the gel. Also note that the instantaneous hoop stress (23) on the edge of the gel is tensile.

We next consider the long-time limit, $t \rightarrow \infty$. After being compressed for a long time, the gel reaches a new state of equilibrium: the chemical potential of the solvent everywhere in the gel equals that in the external solvent, $\mu(r, \infty)$ $=0$. The radial and hoop stresses vanish, $\sigma_{r}(r, \infty)=\sigma_{\theta}(r, \infty)$ $=0$. From Eqs. (8) and (9), we obtain the radial and the hoop strains:

$$
\varepsilon_{r}(r, \infty)=\varepsilon_{\theta}(r, \infty)=\nu \varepsilon .
$$

The radial displacement is

$$
u(r, \infty)=\nu \varepsilon r .
$$

Equation (10) gives the axial stress

$$
\sigma_{z}(r, \infty)=-2(1+\nu) G \varepsilon .
$$

A comparison of (18) and (25) shows that, as the solvent migrates out the gel, the transverse expansion reduces from the instantaneous value $\varepsilon_{r}(r, 0)=\varepsilon_{\theta}(r, 0)=\varepsilon / 2$, and approaches the value of a new state of equilibrium, $\varepsilon_{r}(r, \infty)$ $=\varepsilon_{\theta}(r, \infty)=\nu \varepsilon$. Thus, Poisson's ratio characterizes the chemomechanical interaction of the gel. Poisson's ratio is restricted in the interval $-1<\nu<1 / 2$ by the requirement that the free-energy density is positive definite. When a gel is subject to compression and reaches a new state of equilib- 


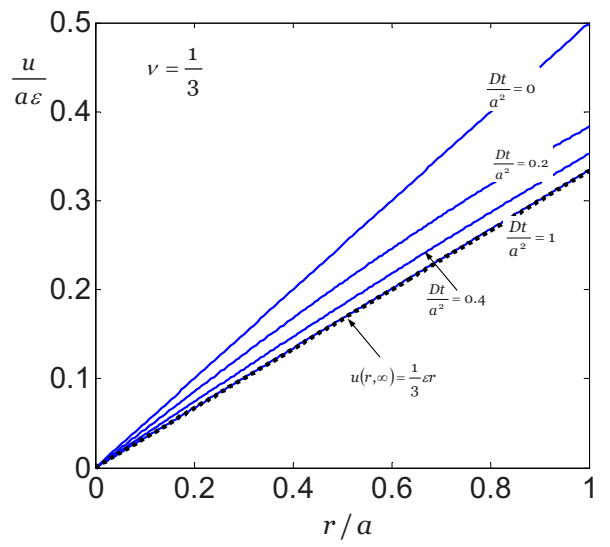

FIG. 2. (Color online) The distribution of the radial displacement at several times.

rium with the external solvent, no solvent in the gel migrates out if $\nu \rightarrow 1 / 2$, or portion of the solvent in the gel migrates out if $-1<\nu<1 / 2$.

\section{TRANSIENT FIELDS}

The partial differential Eqs. (11)-(13), along with the boundary conditions (15) and (16) and the initial conditions (19) and (20), can be solved by the method of separation of variables. Consider displacement fields of,

$$
u(r, t)=\nu \varepsilon r+f(r) \exp (-\alpha t) .
$$

The first term is the long-time limit, and the second term represents the transient deviation from the new state of equilibrium. Inserting (28) into (11) and (13), we obtain that

$$
\frac{D d}{d r}\left[\frac{d(r f)}{r d r}\right]+\alpha f=0, \quad 0<r<a .
$$

The solution to this ordinary differential equation is the Bessel functions. Let $J_{m}(\xi)$ be the Bessel function of order $m$. The displacement field that solves (11)-(13), (15), (16), (19), and (20) is

$$
\frac{u(r, t)}{a \varepsilon}=\nu\left(\frac{r}{a}\right)+\sum_{n=1}^{\infty} B_{n} J_{1}\left(\lambda_{n} \frac{r}{a}\right) \exp \left(-\lambda_{n}^{2} \frac{D t}{a^{2}}\right) .
$$

The eigenvalues $\lambda_{n}$ are determined by (17), namely,

$$
(1-\nu) \lambda_{n} J_{1}^{\prime}\left(\lambda_{n}\right)+\nu J_{1}\left(\lambda_{n}\right)=0 .
$$

The coefficients $B_{n}$ are determined by the initial condition (19), giving

$$
B_{n}=\frac{(1-2 \nu)(1-\nu)^{2} \lambda_{n}}{\lambda_{n}^{2}(1-\nu)^{2}-(1-2 \nu)} \frac{J_{2}\left(\lambda_{n}\right)}{J_{1}^{2}\left(\lambda_{n}\right)} .
$$

Figure 2 plots the displacement field at several times. Instantaneously after the gel is compressed, at $D t / a^{2}=0$, the disk expands in the radial direction, and the displacement is linear in the radius, $u(r, 0)=\varepsilon r / 2$. As time proceeds, solvent gradually migrates out the gel, and the disk shrinks. When $D t / a^{2}$ $\rightarrow \infty$, the gel attains the new state of equilibrium, $u(r, 0)$ $=\nu \varepsilon r$. Figure 2 indicates that the gel nearly attains the new state of equilibrium when $D t / a^{2}=1$.

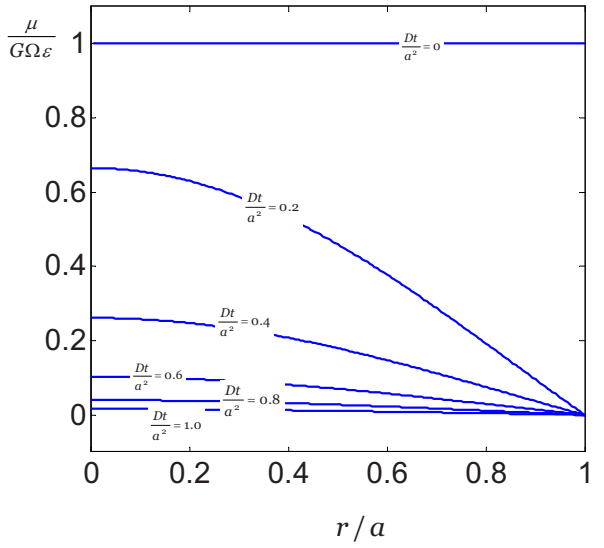

FIG. 3. (Color online) The distribution of the chemical potential of the solvent in the disk at several times.

Inserting the displacement field (30) into (12), and using the boundary condition $\mu(a, t)=0$, we obtain the field of chemical potential:

$$
\begin{aligned}
\frac{\mu(r, t)}{G \Omega \varepsilon}= & \frac{2(1-\nu)}{1-2 \nu} \sum_{n=1}^{\infty} B_{n} \lambda_{n}\left[J_{0}\left(\lambda_{n} \frac{r}{a}\right)-J_{0}\left(\lambda_{n}\right)\right] \\
& \times \exp \left(-\lambda_{n}^{2} \frac{D t}{a^{2}}\right) .
\end{aligned}
$$

Figure 3 plots the chemical potential field at several times. Immediately after compression, the chemical potential of the solvent inside the gel is homogenous, $\mu(r, 0)=\varepsilon G \Omega$. This chemical potential exceeds the chemical potential of solvent outside the gel, $\mu=0$, and drives the solvent to migrate out. The chemical potential of the solvent in the gel at the edge of the disk is taken to equal that in the external solvent at all time, $\mu(a, t)=0$. As time proceeds, the chemical potential of the solvent in the gel gradually decreases. In the long-time limit, the compressed gel equilibrates with the external solvent, and the chemical potential of the solvent in the gel approaches zero.

Inserting the displacement field (30) and the chemical potential field (33) into the equations of state (8)-(10), we obtain the stresses:

$$
\begin{aligned}
\frac{\sigma_{\theta}}{G \varepsilon}= & \sum_{n=1} 2 B_{n}\left\{\frac{a}{r} J_{1}\left(\lambda_{n} \frac{r}{a}\right)+\lambda_{n}\left[-J_{0}\left(\lambda_{n} \frac{r}{a}\right)\right.\right. \\
& \left.\left.+\frac{1-\nu}{1-2 \nu} J_{0}\left(\lambda_{n}\right)\right]\right\} \exp \left(-\lambda_{n}^{2} \frac{D t}{a^{2}}\right), \\
\frac{\sigma_{r}}{G \varepsilon}= & \sum_{n=1} 2 B_{n}\left[-\frac{a}{r} J_{1}\left(\lambda_{n} \frac{r}{a}\right)+\frac{1-\nu}{1-2 \nu} \lambda_{n} J_{0}\left(\lambda_{n}\right)\right] \\
& \times \exp \left(-\lambda_{n}^{2} \frac{D t}{a^{2}}\right), \\
\frac{\sigma_{z}}{G \varepsilon}= & -2(\nu+1)+\sum_{n=1} 2 B_{n} \lambda_{n}\left[-J_{0}\left(\lambda_{n} \frac{r}{a}\right)\right. \\
& \left.+\frac{1-\nu}{1-2 \nu} J_{0}\left(\lambda_{n}\right)\right] \exp \left(-\lambda_{n}^{2} \frac{D t}{a^{2}}\right) .
\end{aligned}
$$



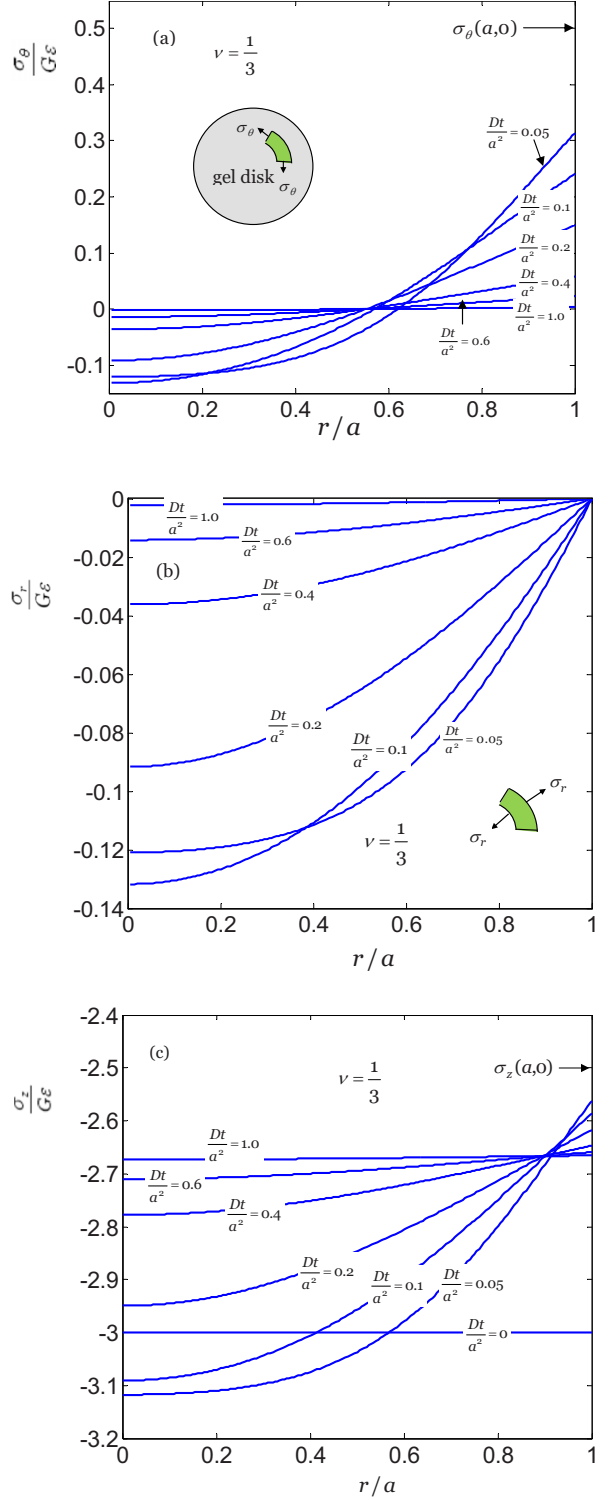

FIG. 4. (Color online) The evolution of (a) the hoop stress, (b) the radial stress, and (c) the axial stress.

Figure 4(a) plots the distribution of the hoop stress at several times. Instantaneously after compression, the solvent in the gel has no time to migrate, so that no hoop stress develops in the interior of the disk. However, a tensile hoop stress develops instantaneously at the edge of the disk, as discussed before. After the gel is compressed for some time, solvent leaves the gel gradually, so that the concentration becomes inhomogeneous: the concentration of the solvent near the edge is lower than that around the center. As time proceeds, the tensile stress reduces magnitude but spreads over a larger region. Toward the center of the disk, the hoop stress is compressive. In the long-time limit, the hoop stress everywhere in the gel vanishes.

Figure 4(b) plots the distribution of the radial stress at several times. The radial stress at the edge vanishes at all time, as dictated by the boundary condition. After the gel is compressed by the plates, the solvent migrates out, initially from the region near the edge of the disk. Consequently, the radial stress around the center of the disk is compressive.

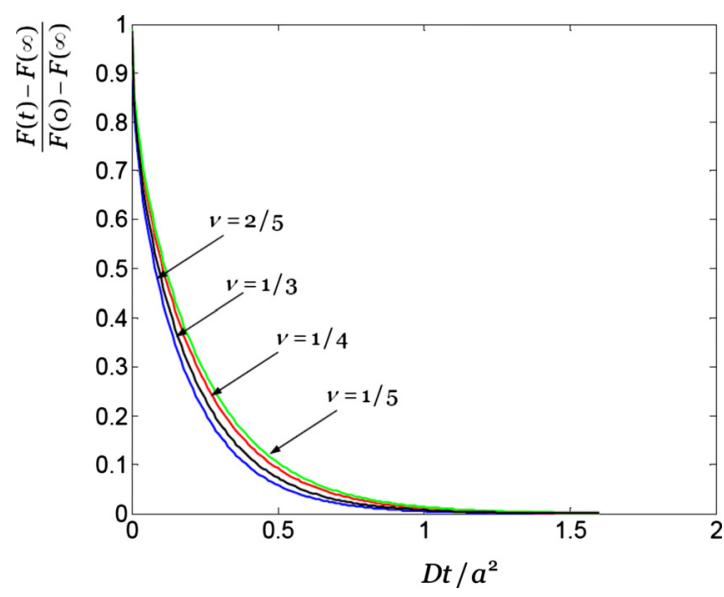

FIG. 5. (Color online) The compressive force relaxes as a function of time. The relaxation curve varies with Poisson's ratio somewhat.

The magnitude of the compressive radial stress initially rises and then falls. In the long-time limit, the radial stress everywhere vanishes.

Figure 4(c) plots the distribution of the axial stress at several times. As discussed before, instantaneously after the gel is pressed, the axial stress is $\sigma_{z}(a, 0)=-G \varepsilon(2-\nu) /(1$ $-\nu)$ at the edge of the disk, and is $\sigma_{z}(r, 0)=-3 G \varepsilon$ in the interior of the disk. These two levels of the axial stress are unequal, so long as $\nu \neq 0.5$. The magnitude of the axial stress at the edge of the disk increases as time progresses. The magnitude of the axial stress at the center of the disk initially rises and then falls. After some time, the axial stress homogenizes in the disk, and approaches the long-time limit $\sigma_{z}(r, \infty)=-2(1+\nu) G \varepsilon$.

\section{USING RELAXATION CURVES TO DETERMINE PROPERTIES OF GELS}

Integrating the axial stress over the area of the disk, we obtain the axial force as a function of time:

$$
\begin{aligned}
\frac{F(t)}{G \varepsilon \pi a^{2}}= & 2(\nu+1)-\sum_{n=1}^{\infty} B_{n}\left(-4 J_{1}\left(\lambda_{n}\right)\right. \\
& \left.+\frac{2(1-\nu)}{1-2 \nu} \lambda_{n} J_{0}\left(\lambda_{n}\right)\right) \exp \left(-\lambda_{n}^{2} \frac{D t}{a^{2}}\right) .
\end{aligned}
$$

The short-time limit is

$$
F(0)=3 \pi G \varepsilon a^{2} .
$$

The long-time limit is

$$
F(\infty)=2 \pi(1+\nu) G \varepsilon a^{2} .
$$

Figure 5 plots the relaxation curve (37) in the form

$$
\frac{F(t)-F(\infty)}{F(0)-F(\infty)}=f\left(\nu, \frac{D t}{a^{2}}\right),
$$

The ratio on the left-hand side measures how far the gel is away from the state of equilibrium. The ratio depends on Poison's ratio weakly, as indicated in Fig. 5.

Covalently crosslinked alginate hydrogels are prepared following the protocol previously described. ${ }^{21}$ The gel is sub- 

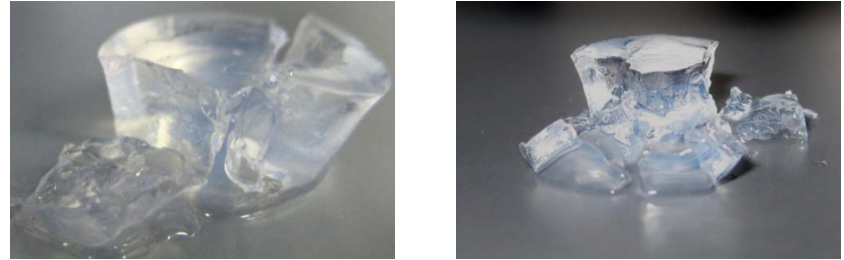

FIG. 6. (Color online) Photos of fractured alginate hydrogel caused by compression.

merged in distilled water for $24 \mathrm{~h}$ until it is fully swollen. From a piece of the gel, we punch out three disks of radii 3, 4 , and $5 \mathrm{~mm}$. These disks are then pressed with a stainless steel plate. The plate first approaches the surface of the disk with a speed of $2 \mu \mathrm{m} / \mathrm{s}$ until the measured force starts to increase. At this point, the gap between the top and bottom plates is viewed as the thickness of the disk, which is 7.71, $7.82,7.65 \mathrm{~mm}$, of $3 \mathrm{~mm}$ radius, $4 \mathrm{~mm}$ radius, and $5 \mathrm{~mm}$ radius sample, respectively. Each disk is subject to a $20 \%$ vertical compressive strain. The total rising time is about 10 $\mathrm{s}$, which is negligible compared to the relaxation time (about 3 to $8 \mathrm{~h}$ ). While the vertical strain is held at the fixed value, the force on the plate is recorded as a function of time by using the AR rheometer from TA Instruments. The resolution of the force is $0.005 \mathrm{~N}$, and data are taken at the rate of 360 points per second.

The covalently crosslinked alginate hydrogels are quite brittle, and sometimes fracture during the experiment (Fig. $6)$. The fracture mechanics of gels is interesting in its own right but will not be pursued in this paper. The data reported below are taken from experiments in which no fracture is observed.

Figure 7(a) plots the relaxation curves measured experimentally from the three disks. In each case, the force rises sharply as the plate is pressed. Subsequently, the plate is held at the fixed position, while the force relaxes and approaches a new state of equilibrium. The magnitude of the force, as well as the relaxation time, is larger when the radius of the disk is larger. Once the force is divided by the area of the disk $\pi a^{2}$, and the time is divided by $a^{2}$, the relaxation curves measured from the disks of the three radii collapse into a single curve [Fig. 7(b)]. This behavior is consistent with the prediction of the theory of poroelasticity. The nominal stress - the force divided by the area of the disk-relaxes as the solvent migrates out from the edge of the gel. The relaxation time is proportional to the radius of the disk squared.

By comparing the relaxation curve measured experimentally with that derived from the theory of poroelasticity, we can determine the shear modulus, Poisson's ratio, and the diffusivity. In the short-time limit, a comparison of the experimental data $F(0) / \pi a^{2}=20.5 \mathrm{kPa}$ and the theoretical formula $F(0) / \pi a^{2}=3 G \varepsilon$ gives the shear modulus $G$ $=34.2 \mathrm{kPa}$. In the long-time limit, a comparison of the experimental data $F(\infty) / F(0)=0.82$ and the theoretical formula $F(\infty) / F(0)=2(1+\nu) / 3$ gives Poisson's ratio $\nu=0.23$. The relaxation curve calculated from the theory of poroelasticity overlaps with the relaxation curves experimentally measured from the three disks when the diffusivity is fit to $D=6.2$ $\times 10^{-9} \mathrm{~m}^{2} / \mathrm{s}$ [Fig. 7(b)].
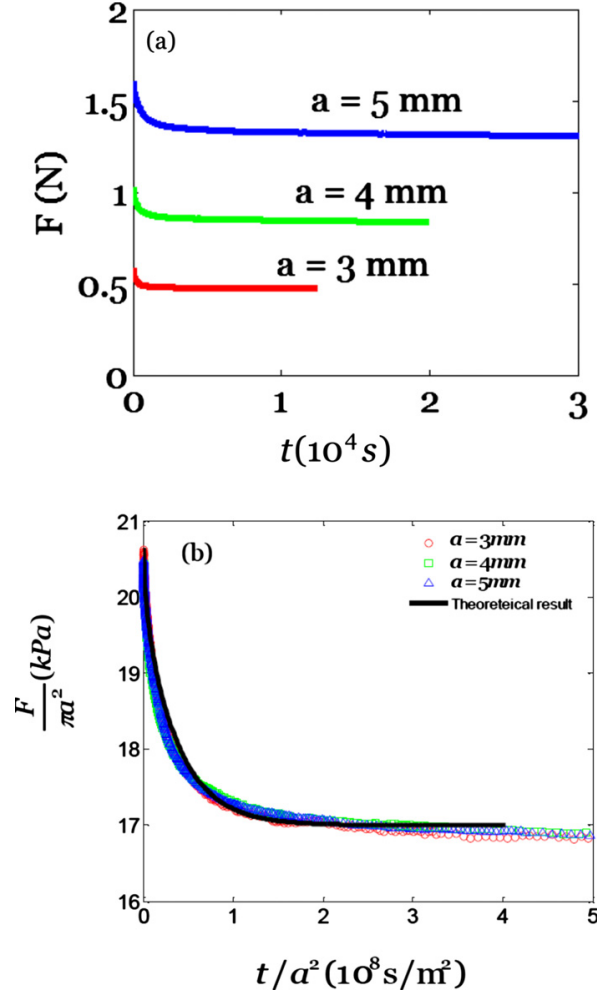

FIG. 7. (Color online) A disk of an alginate hydrogel is compressed between parallel plates, while the force on the planes is recorded as a function of time. (a) Relaxation curves obtained by using disks of an alginate hydrogel of three radii. (b) Each of the three relaxations curves is plotted again, with the force divided by the area of the disk, and the time divided by the radius squared. Also plotted is the relaxation curve obtained from the theory of poroelasticity.

In a recent paper, ${ }^{17}$ we have used a conical indenter to characterize the alginate hydrogel. As illustrated in Fig. 1, the gel is submerged in water and is fully swollen. The conical indenter, of half included angle $\theta$, is suddenly pressed into the gel and is subsequently held at a fixed depth $h$. The force on the indenter is measured as a function of time. This test has been analyzed within the theory of poroelasticity, ${ }^{17}$ and the relevant results are summarized here. The radius of contact is given by

$$
a=\frac{2}{\pi} h \tan \theta .
$$

In the short-time limit, solvent in the gel has no time to migrate, the gel behaves like an incompressible elastic solid, and the force on the indenter is given by

$$
F(0)=4 G a h
$$

In the long-time limit, portion of the solvent in the gel has migrated out, the gel has attained a new state of equilibrium with the external solvent, and the force on the indenter is given by

$$
F(\infty)=2 G a h /(1-\nu) .
$$

For the gel to evolve from the short-time limit toward the long-time limit, the solvent in the gel under the indenter must migrate. The relevant length in this diffusion-type problem is 

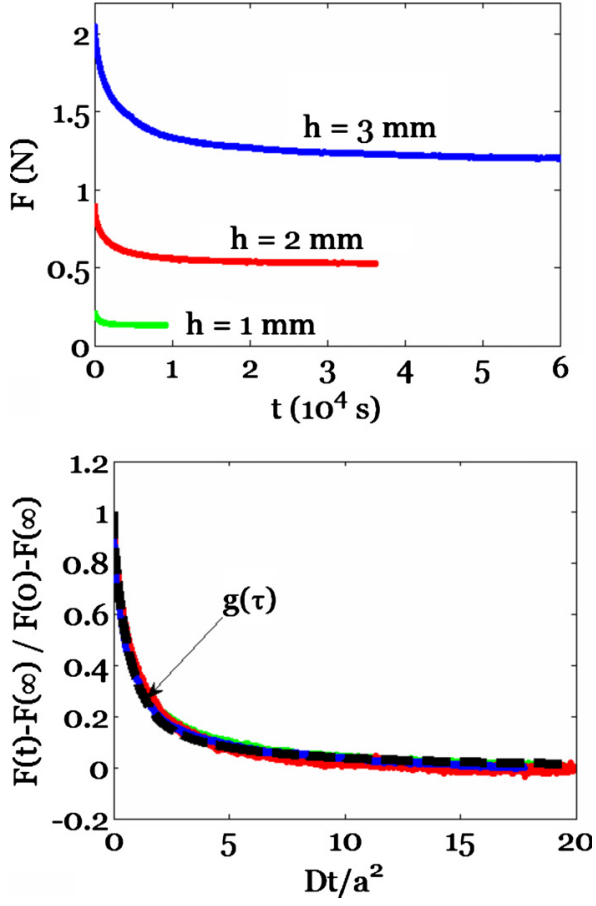

FIG. 8. (Color online) A conical indenter is pressed into a disk of an alginate hydrogel to a certain depth, while the force on the indenter is recorded as a function of time. (a) Relaxation curves obtained by keeping the indenter at three depths. (b) The relaxation curves are plotted again by using normalized variables. Also included is the relaxation curve obtained from the theory of poroelasticity.

the radius of contact, $a$, and the normalized time takes the form $\tau=D t / a^{2}$. The function $F(t)$ obeys

$$
\frac{F(t)-F(\infty)}{F(0)-F(\infty)}=g(\tau)
$$

The dimensionless ratio on the left-hand side of (44) is a measure of how far the gel is away from the new state of equilibrium. The function $g(\tau)$ is determined by solving the poroelastic boundary-value problem. Our previous work indicates that $g$ is a function of the single variable $\tau$, given by

$$
g(\tau)=0.493 \exp (-0.822 \sqrt{\tau})+0.507 \exp (-1.348 \tau) .
$$

To minimize the variability of the material, we make the alginate hydrogels for both tests-compression and indentation - in the same batch. The solutions are poured into a plastic mold of $3 \mathrm{~cm}$ radius and $2 \mathrm{~cm}$ thickness. After gelation, the gel is submerged in distilled water for $48 \mathrm{~h}$ until it is fully swollen. We then press an aluminum indenter of half included angle $\theta=70^{\circ}$ into the gel to a fixed depth. The force on the indenter is recorded as a function of time by using a custom-built load frame with a force resolution of $0.01 \mathrm{~N}$ and a displacement resolution of $1 \mu \mathrm{m}$. The indenters are programmed to approach the surface of the sample at the speed of $2 \mu \mathrm{m} / \mathrm{s}$, until the slope of the recorded forcedisplacement curve start to be positive. The time used to press the indenter into the alginate gels $(10 \mathrm{~s})$ is much shorter than the relaxation time ( 3 to $16 \mathrm{~h}$ ), so that the effect of the initial loading stage is minimized.

Figure 8(a) shows the measured relaxation curves re- corded at the three depths of indentation. In each case, the force rises sharply, and then relaxes as the gel approaches a new state of equilibrium with the external solvent. The magnitude of the force, as well as the relaxation time, is larger when the depth of indentation is larger. A comparison of the experimental value $F(0) / a h=130 \mathrm{kPa}$ and the analytical formula $F(0) / a h=4 G$ gives $G=32.5 \mathrm{kPa}$. A comparison of the experimental value $F(0) / F(\infty)=1.56$ and the analytical formula $F(0) / F(\infty)=2(1-\nu)$ gives Poisson's ratio $\nu=0.22$.

Figure $8(\mathrm{~b})$ plots the relaxation curves measured with the three depths indentation the in a dimensionless form. The three curves collapse into a single curve. Furthermore, these curves overlap with the relaxation curve calculated with the theory of poroelasticity, $g(\tau)$ in (45), when the diffusivity is fit to the value $D=6.6 \times 10^{-9} \mathrm{~m}^{2} / \mathrm{s}$.

Comparing the material properties measured by compression and indentation, we note $5.2 \%$ difference in the shear modulus, $4.6 \%$ difference in Poisson's ration, and $6 \%$ in the diffusivity. This excellent agreement lends support to both tests. The two tests have their own advantages and disadvantages. The compression test requires the sample to be fabricated with perfectly parallel top and bottom surfaces, which may be difficult in practice. This concern is absent for the indentation test because the starting point of the measurement is readily detected for conical or spherical indenters. The indentation test, however, requires the thickness of the sample to be more than ten times larger than the depth of indentation. This requirement might be difficult to satisfy in practice.

\section{CONCLUDING REMARKS}

The compression test is analyzed within the theory of poroelasticity. By comparing the relaxation curve derived from the theory to that measured in the experiment, we obtain the shear modulus, Poisson's ratio and the permittivity of a covalently crosslinked alginate hydrogel. The material constants so determined agree well with those obtained from a recently developed indentation method. The agreement lends support to both methods. Furthermore, our calculation shows that, as the compressed gel relaxes, the concentration of the solvent in the gel is inhomogeneous, resulting in tensile hoop stresses near the edge of the gel. While fracture is indeed often observed in our experiments, the mechanics of fracture awaits clarification.

\section{ACKNOWLEDGMENTS}

This work is supported by the NSF (Grant No. CMMI0800161) and by the MRSEC at Harvard University.

${ }^{1}$ R. Duncan, Nat. Rev. Drug Discovery 2, 347 (2003).

${ }^{2}$ N. A. Peppas, J. Z. Hilt, A. Khademhosseini, and R. Langer, Adv. Mater. 18, 1345 (2006)

${ }^{3}$ A. Richter, G. Paschew, S. Klatt, J. Lienig, K. Arndt, and H. P. Adler, Sensors 8, 561 (2008).

${ }^{4}$ P. Calvert, Adv. Mater. 21, 743 (2009).

${ }^{5}$ K. Y. Lee and D. J. Mooney, Chem. Rev. 101, 1869 (2001).

${ }^{6}$ S. Varghese and J. H. Elisseeff, Adv. Polym. Sci. 203, 95 (2006).

${ }^{7}$ M. Kleverlaan, R. H. van Hoort, and I. Jones, SPE/IADC Drilling Conference, Amsterdam, Netherlands, 23-25 February, 2005 (Society of Petroleum Engineers, Richardson, TX, 2005). 
${ }^{8}$ S. Q. Cai, Y. Lou, P. Ganguly, A. Robisson, and Z. G. Suo, J. Appl. Phys. 107, 103535 (2010).

${ }^{9}$ L. Barrangou, C. Daubert, and E. Foegeding, Food Hydrocolloids 20, 184 (2006).

${ }^{10}$ J. Zhang, C. Daubert, and E. Foegeding, J. Food Sci. 70, e425 (2005).

${ }^{11}$ K. Urayama, Y. Taoka, K. Nakamura, and T. Takigawa, Polymer 49, 3295 (2008).

${ }^{12}$ C. Y. Hui, Y. Y. Lin, F. C. Chuang, K. R. Shull, and W. C. Ling, J. Polym. Sci., Part B: Polym. Phys. 44, 359 (2006).

${ }^{13}$ M. L. Oyen, J. Mater. Res. 23, 1307 (2008).

${ }^{14}$ V. Morel and T. Quinn, J. Orthop. Res. 22, 145 (2004).

${ }^{15}$ B. Kurz, M. Jin, P. Patwari, D. Cheng, M. Lark, and A. Grodinsky, J.
Orthop. Res. 19, 1140 (2001).

${ }^{16}$ X. H. Zhao, N. D. Huebsch, D. J. Mooney, and Z. G. Suo, J. Appl. Phys. 107, 063509 (2010)

${ }^{17}$ Y. Hu, X. Zhao, J. Vlassak, and Z. Suo, Appl. Phys. Lett. 96, 121904 (2010).

${ }^{18}$ M. A. Biot, J. Appl. Phys. 12, 155 (1941).

${ }^{19}$ M. Doi, J. Phys. Soc. Jpn. 78, 052001 (2009).

${ }^{20}$ J. Yoon, S. Q. Cai, Z. G. Suo, and R. C. Hayward, "Poroelastic swelling kinetics of thin hydrogel layers: Comparison of theory and experiment," Soft Matter (to be published).

${ }^{21}$ K. H. Bouhadir, D. S. Hausman, and D. J. Mooney, Polymer 40, 3575 (1999). 